EUROPEAN LABORATORY FOR PARTICLE PHYSICS

CERN-EP-042

14 March 2000

\title{
Search for eV (pseudo)scalar penetrating particles in the SPS neutrino beam
}

\section{NOMAD Collaboration}

P. Astier ${ }^{n}$ D. Autiero ${ }^{\text {h A. Baldisseri }}{ }^{\mathrm{s}}$ M. Baldo-Ceolin ${ }^{\mathrm{m}}$ G. Ballocchi ${ }^{\text {h }}$ M. Banner ${ }^{\mathrm{n}}$ G. Bassompierre ${ }^{\mathrm{a}}$ K. Benslama ${ }^{\mathrm{i}}$ N. Besson ${ }^{\mathrm{s}}$ I. Bird ${ }^{\mathrm{h}, \mathrm{i}}$ B. Blumenfeld ${ }^{\mathrm{b}}$ F. Bobisut ${ }^{\mathrm{m}}$ J. Bouchez ${ }^{\mathrm{s}}$ S. Boyd ${ }^{\mathrm{v}}$ A. Bueno ${ }^{\mathrm{c}, \mathrm{z}}$ S. Bunyatov ${ }^{\mathrm{f}}$ L. Camilleri $^{\mathrm{h}}$ A. Cardini ${ }^{\mathrm{j}}$ P.W. Cattaneo ${ }^{\circ}$ V. Cavasinni ${ }^{\mathrm{q}}$ A. Cervera-Villanueva ${ }^{\mathrm{h}, \mathrm{x}}$ G. Collazuol ${ }^{\mathrm{m}}$ G. Conforto ${ }^{\mathrm{h}, \mathrm{w}}$ C. Conta $^{\mathrm{o}}$ M. Contalbrigo ${ }^{\mathrm{m}}$ R. Cousins ${ }^{j}$ D. Daniels ${ }^{c}$ H. Degaudenzi ${ }^{i}$ T. Del Prete ${ }^{q}$ A. De Santo ${ }^{\text {h,q }}$ T. Dignan ${ }^{c}$ L. Di Lella ${ }^{\text {h }}$ E. do Couto e Silva ${ }^{h}$ J. Dumarchez ${ }^{n}$ M. Ellis ${ }^{v}$ G.J. Feldman ${ }^{c}$ R. Ferrari ${ }^{\circ}$ D. Ferrère ${ }^{\mathrm{h}}$ V. Flaminio ${ }^{\mathrm{q}}$ M. Fraternali ${ }^{\circ}$ J.-M. Gaillard ${ }^{\mathrm{a}}$ E. Gangler ${ }^{\mathrm{h}, \mathrm{n}}$ A. Geiser ${ }^{\mathrm{e}, \mathrm{h}}$ D. Geppert ${ }^{\mathrm{e}}$ D. Gibin ${ }^{\mathrm{m}}$ S.N. Gninenko ${ }^{\mathrm{h}, \ell}$ A. Godley ${ }^{\mathrm{v}}$ J.-J. Gomez-Cadenas ${ }^{\mathrm{h}, \mathrm{x}}$ J. Gosset ${ }^{\mathrm{s}}$ C. Gößling ${ }^{\mathrm{e}} \mathrm{M}$. Gouanère ${ }^{\mathrm{a}} \mathrm{A}$. Grant ${ }^{\mathrm{h}}$ G. Graziani ${ }^{\mathrm{g}}$ A. Guglielmi ${ }^{m}$ C. Hagner ${ }^{\mathrm{s}}$ J. Hernando ${ }^{\mathrm{x}}$ D. Hubbard ${ }^{\mathrm{c}}$ P. Hurst ${ }^{c}$ N. Hyett ${ }^{k}$ E. Iacopini ${ }^{g}$ C. Joseph ${ }^{i}$ F. Juget ${ }^{i}$ M.M. Kirsanov ${ }^{\ell}$ O. Klimov ${ }^{f}$ J. Kokkonen ${ }^{\text {h }}$ A.V. Kovzelev ${ }^{\ell, o}$ N.V. Krasnikov ${ }^{\ell}$ A. Krasnoperov ${ }^{\text {a,f }}$ V.E. Kuznetsov ${ }^{\text {f,h }}$ S. Lacaprara ${ }^{\mathrm{m}}$ C. Lachaud $^{\mathrm{n}}$ B. Lakić ${ }^{\mathrm{y}}$ A. Lanza $^{\mathrm{O}}$ L. La Rotonda ${ }^{\mathrm{d}}$ M. Laveder ${ }^{\mathrm{m}}$ A. Letessier-Selvon ${ }^{\mathrm{n}}$ J.-M. Levy $^{\mathrm{n}}$ L. Linssen ${ }^{\text {h }}$ A. Ljubičić ${ }^{y}$ J. Long ${ }^{b}$ A. Lupi ${ }^{g}$ A. Marchionni ${ }^{g}$ F. Martelli ${ }^{\mathrm{w}}$ X. Méchain ${ }^{\mathrm{s}}$ J.-P. Mendiburu ${ }^{\mathrm{a}}$ J.-P. Meyer ${ }^{\mathrm{s}}$ M. Mezzetto ${ }^{\mathrm{m}}$ S.R. Mishra ${ }^{\text {c,u }}$ G.F. Moorhead ${ }^{\mathrm{k}}$ L. Mossuz $^{\mathrm{a}}$ P. Nédélec ${ }^{\mathrm{a}} \mathrm{Yu}$. Nefedov ${ }^{\mathrm{f}}$ C. Nguyen-Mau ${ }^{\mathrm{i}}$ D. Orestano ${ }^{\mathrm{r}}$ F. Pastore ${ }^{\mathrm{r}}$ L.S. Peak ${ }^{\mathrm{v}}$ E. Pennacchio ${ }^{\mathrm{w}}$ H. Pessard ${ }^{\mathrm{a}}$ R. Petti ${ }^{\mathrm{o}}$ A. Placci ${ }^{\text {h }}$ G. Polesello ${ }^{\circ}$ D. Pollmann ${ }^{\text {e }}$ A.Yu. Polyarush ${ }^{\ell}$ B. Popov ${ }^{f, n}$ C. Poulsen ${ }^{k}$ J. Rico ${ }^{z}$ C. Roda ${ }^{\text {h,q }}$ A. Rubbia ${ }^{\text {h,z }}$ 
F. Salvatore ${ }^{o}$ K. Schahmaneche ${ }^{n}$ B. Schmidt ${ }^{e, h}$ T. Schmidt ${ }^{e}$ M. Sevior ${ }^{k}$ D. Sillou ${ }^{a}$ F.J.P. Soler ${ }^{h, v}$ G. Sozzi ${ }^{i}$ D. Steele ${ }^{b, i}$ M. Steininger ${ }^{i}$ U. Stiegler ${ }^{h}$ M. Stipčević ${ }^{y}$ Th. Stolarczyk ${ }^{\mathrm{s}}$ M. Tareb-Reyes ${ }^{i}$ G.N. Taylor ${ }^{k}$ V. Tereshchenko ${ }^{\text {f A.N. Toropin }}{ }^{\ell}$ A.-M. Touchard ${ }^{n}$ S.N. Tovey ${ }^{\mathrm{h}, \mathrm{k}}$ M.-T. Tran ${ }^{\mathrm{i}}$ E. Tsesmelis ${ }^{\mathrm{h}}$ J. Ulrichs ${ }^{\mathrm{v}}$ L. Vacavant ${ }^{\mathrm{i}}$ M. Valdata-Nappi ${ }^{\mathrm{d}, \mathrm{p}}$ V. Valuev ${ }^{\mathrm{f}, \mathrm{j}}$ F. Vannucci ${ }^{\mathrm{n}}$ K.E. Varvell ${ }^{\mathrm{v}}$ M. Veltri ${ }^{\mathrm{w}}$ V. Vercesi ${ }^{\mathrm{o}}$ D. Verkindt ${ }^{a}$ J.-M. Vieira ${ }^{i}$ T. Vinogradova ${ }^{j}$ S.A. Volkov ${ }^{\ell}$ F.V. Weber ${ }^{\mathrm{c}, \mathrm{h}}$ T. Weisse ${ }^{\mathrm{e}}$ F.F. Wilson ${ }^{\mathrm{h}}$ L.J. Winton ${ }^{\mathrm{k}}$ B.D. Yabsley ${ }^{\mathrm{v}}$ H. Zaccone ${ }^{\mathrm{s}}$ K. Zioutas ${ }^{\mathrm{h}}$ K. Zuber ${ }^{\mathrm{e}}$ P. Zuccon ${ }^{\mathrm{m}}$ ${ }^{\mathrm{a}}$ LAPP, Annecy, France

${ }^{\mathrm{b}}$ Johns Hopkins Univ., Baltimore, MD, USA

${ }^{\mathrm{c}}$ Harvard Univ., Cambridge, MA, USA

${ }^{\mathrm{d}}$ Univ. of Calabria and INFN, Cosenza, Italy

${ }^{\mathrm{e}}$ Dortmund Univ., Dortmund, Germany

${ }^{\mathrm{f}}$ JINR, Dubna, Russia

${ }^{\mathrm{g}}$ Univ. of Florence and INFN, Florence, Italy

${ }^{\mathrm{h}} C E R N$, Geneva, Switzerland

${ }^{\mathrm{i}}$ University of Lausanne, Lausanne, Switzerland

${ }^{j} U C L A$, Los Angeles, CA, USA

${ }^{\mathrm{k}}$ University of Melbourne, Melbourne, Australia

${ }^{\ell}$ Inst. Nucl. Research, INR Moscow, Russia

${ }^{\mathrm{m}}$ Univ. of Padova and INFN, Padova, Italy

${ }^{\mathrm{n}}$ LPNHE, Univ. of Paris VI and VII, Paris, France

${ }^{\circ}$ Univ. of Pavia and INFN, Pavia, Italy

${ }^{\mathrm{p}}$ Univ. of Perugia and INFN, Perugia, Italy

${ }^{\mathrm{q}}$ Univ. of Pisa and INFN, Pisa, Italy

${ }^{\mathrm{r}}$ Roma Tre University and INFN, Rome, Italy

${ }^{\mathrm{S}}$ DAPNIA, CEA Saclay, France

${ }^{\mathrm{t}}$ ANSTO Sydney, Menai, Australia

${ }^{\mathrm{u}}$ Univ. of South Carolina, Columbia, SC, USA

${ }^{\mathrm{v}}$ Univ. of Sydney, Sydney, Australia

${ }^{\mathrm{w}}$ Univ. of Urbino, Urbino, and INFN Florence, Italy

xIFIC, Valencia, Spain

${ }^{\mathrm{y}}$ Rudjer Bošković Institute, Zagreb, Croatia

${ }^{\mathrm{z} E T H ~ Z u ̈ r i c h, ~ Z u ̈ r i c h, ~ S w i t z e r l a n d ~}$ 


\begin{abstract}
We carried out a model-independent search for light scalar or pseudoscalar particles a's (an example of which is the axion) that couple to two photons by using a photon-regeneration method at high energies allowing a substantial increase in the sensitivity to $\mathrm{eV}$ masses. The experimental set-up is based on elements of the CERN West Area Neutrino Facility (WANF) beam line and the NOMAD neutrino detector. The new particles, if they exist, could be produced through the Primakoff effect in interactions of high energy photons, generated by the $450 \mathrm{GeV}$ protons in the CERN SPS neutrino target, with virtual photons from the WANF horn magnetic field. The particles would penetrate the downstream shielding and would be observed in the NOMAD neutrino detector through their re-conversion into real high energy photons by interacting with the virtual photons from the magnetic field of the NOMAD dipole magnet.

From the analysis of the data collected during the 1996 run with $1.08 \times 10^{19}$ protons on target, 312 candidate events with energy between 5 and $140 \mathrm{GeV}$ were found. This number is in general agreement with the expectation of $272 \pm 18$ background events from standard neutrino processes. A $90 \% C L$ upper limit on the $a \gamma \gamma$-coupling $g_{a \gamma \gamma}<1.5 \times 10^{-4} \mathrm{GeV}^{-1}$ for $a$ masses up to $40 \mathrm{eV}$ is obtained.
\end{abstract}

Key words: light (pseudo)scalars, photon-regeneration

\title{
1 Introduction
}

Neutral spin-zero scalar or pseudoscalar particles $a$ 's (this notation will be used for both cases) of nearly zero mass are predicted in many theories. The most motivated pseudo-Nambu-Goldstone bosons, for example, arise in models constraining spontaneously broken symmetry, see e.g. [1]. They couple to the divergence of the current whose charge generates the symmetry which is spontaneously broken. The most popular light pseudoscalar, the axion, postulated [2] to provide a solution of the "strong CP" problem, emerges as a consequence of the breaking of the Peccei-Quinn symmetry [3]. It is now believed that the axion has a mass much smaller than $\sim O(100) k e V$ that was originally expected [4]-[6]. The axion two-photon interaction is given by the Lagrangian

$$
L_{i n t}=-\frac{1}{4} g_{a \gamma \gamma} F_{\mu \nu} \tilde{F}^{\mu \nu} a=g_{a \gamma \gamma}(\mathbf{E} \cdot \mathbf{B}) \cdot a
$$

where $g_{a \gamma \gamma}$ is the coupling constant, E, B are the electric and magnetic fields, 
$a$ is the axion field. If $\mathbf{B}$ is an external magnetic field, $a$ 's interact with the photon electric field component parallel to $\mathbf{B}$.

An example of a scalar particle weakly coupled to two photons is the dilaton, which arises in super-string theories and interacts with matter through the trace of the energy-momentum tensor[7]. In particular its interaction with photons is given by the Lagrangian

$$
L_{a \gamma \gamma}=-\frac{1}{4} g_{a \gamma \gamma} F_{\mu \nu} F^{\mu \nu} a=\frac{1}{2} g_{a \gamma \gamma}\left(\mathbf{B}^{2}-\mathbf{E}^{2}\right) \cdot a
$$

Here again, $g_{a \gamma \gamma}$ is the coupling constant and $a$ is the dilaton field. If $\mathbf{B}$ is an external magnetic field $a$ 's interact with the photon electric field component orthogonal to B. Usually it is assumed that $g_{a \gamma \gamma}=O\left(M_{P l}^{-1}\right)$ and that the dilaton mass $m_{a}=O\left(M_{P l}\right)$, where $M_{P l}$ is the Planck mass. However, in recent models with large compactification radii (see e.g. [8]), the dilaton could be rather light and since there are no firm predictions for the coupling $g_{a \gamma \gamma}$ the searches for such particles become very interesting and actual.

Several experiments have placed limits on possible (pseudo)scalar bosons [1]. Experimental bounds on $g_{a \gamma \gamma}$ for light $a$ 's can be obtained from laser experiments [9], [10], from experiments on $J / \psi$ and $\Upsilon$ particles [11], and from orthopositronium decays [12]. The best direct experimental limit, $g_{a \gamma \gamma}<2 \times$ $10^{-3} \mathrm{GeV}^{-1}$ for the $\mathrm{eV}$ mass range, has been extracted from the recent limit of the CLEO Collaboration $\operatorname{Br}(\Upsilon(1 S) \rightarrow a \gamma)<1.3 \times 10^{-5}$ [13] by assuming this decay to occur through a virtual photon [14].

The best limit on the axion-photon coupling comes from astrophysical limits on anomalous energy loss by stars [15]. However, such astrophysical constraints, although more stringent, are model-dependent and have various uncertainties. For example, as has been demonstrated in [16], the inclusion of additional fermions which strongly interact with (pseudo)scalars of mass $m_{a} \sim O(10) e V$ may evade the astrophysics constraints. Hence, it is important to perform independent laboratory tests on the existence of such particles in the mass range discussed above.

Experimental techniques used for searching for light (pseudo)scalars are based either on the measurement of vacuum birefringence [17]-[19], or on the Helioscope method [20],[21], or on what is called "photon-regeneration" [22]. Here we describe a direct experimental search for $a$ particles which are weakly coupled to two photons and which might be present in the SPS neutrino beam. The experiment is performed by using elements of the CERN West Area Neutrino Facility (WANF) beam line and the NOMAD neutrino detector and is based on the photon-regeneration method, used for the first time at high energy. In the analysis we do not assume any relationship between particle mass and coupling to photons but we assume that $a$ 's are rather long-lived particles. The present analysis as well as the experimental signature of the signal events 
are similar to those of our previous light gauge boson search [23].

\section{WANF and NOMAD detector}

The present WANF beam line [24] (schematically shown in Figure 1) provides an essentially pure $\nu_{\mu}$ beam for the CERN neutrino experiments. It consists of a beryllium target irradiated by $450 \mathrm{GeV}$ protons from the CERN SPS. The secondary hadrons are focused with two magnetic elements, the horn and the reflector, located in front of a $290 \mathrm{~m}$ long vacuum decay tunnel. Protons that have not interacted in the target, secondary hadrons and muons that do not decay are absorbed by a $400 \mathrm{~m}$ shielding made of iron and earth. The NOMAD detector is located at about $835 \mathrm{~m}$ from the neutrino target. The horn operates in pulsed mode and has a length of nearly $7 \mathrm{~m}$. Its magnetic field is transverse to the beam, azimuthally symmetric and its radial dependence is $B[T]=0.02 \times I[k A] / r[\mathrm{~cm}]$ between the inner and outer aluminium conductors, where $I=100 \mathrm{kA}$ is the horn current and $r$ is the distance from the horn axis. At the point where the inner conductor is closest to the axis $(r \simeq 1 \mathrm{~cm})$, a maximum magnetic field of $\sim 2 T$ is achieved. The second focusing element, the reflector, has a maximum field of $\sim 0.2 T$ and its contribution is negligible.

The NOMAD detector is described in Ref. [25]. It consists of a number of sub-detectors most of which are located inside a $0.4 T$ dipole magnet with a volume of $7.5 \times 3.5 \times 3.5 \mathrm{~m}^{3}$ : an active target of drift chambers (DC) [26] with a mass of 2.7 tons (mainly carbon), an average density of $0.1 \mathrm{~g} / \mathrm{cm}^{3}$ and a total length of about one radiation length $\left(\sim 1.0 X_{0}\right)$ followed by a transition radiation detector (TRD) [27], a preshower detector (PRS), and an electromagnetic calorimeter (ECAL). The PRS is composed of a plane of horizontal proportional tubes and one of vertical proportional tubes preceded by a $9 \mathrm{~mm}$ thick lead plate $\left(1.6 X_{0}\right)$. The ECAL consists of 875 lead-glass counters, each of about $19 X_{0}$ deep, arranged in a matrix of 35 rows by 25 columns [28].

A hadron calorimeter (HCAL) and two muon stations are located just after the magnet coils. The trigger for neutrino interactions in the DC target is provided by two planes of scintillation counters $T_{1}$ and $T_{2}$. Interactions in the PRS or ECAL which are used in the present study are collected by the trigger $\overline{T_{1} \times T_{2}} \times E C A L$, referred to as the ECAL trigger [29]. The ECAL signal is obtained as the OR of all counter signals exceeding a threshold of $\sim 0.8 \mathrm{GeV}$. The average rate of ECAL triggers is about 3 for $10^{13}$ protons incident on the neutrino target $(p o t)$. 


\section{Detection Principle}

This experiment was possible due to the unique combination of several factors, namely, the presence of: i) the high $\int B_{\perp} d z$ regions both in the WANF horn and in the NOMAD detector, where $z$ is the coordinate along the magnet axis; ii) the relatively transparent DC target, used to reject efficiently interactions in the magnet coils; iii) the PRS and ECAL to detect photons. The experimental set-up consisting of WANF elements and the NOMAD detector and the detection principle are schematically illustrated in Figure 1.

If $a$ 's exist, one expects a flux of such high energy particles in the SPS neutrino beam because both scalar and pseudoscalar a's could be produced in the forward direction through the Primakoff effect in interactions of high energy photons, generated by $450 \mathrm{GeV}$ protons in the neutrino target, with virtual photons from the WANF horn magnetic field.

If $a$ is a long-lived particle, it would penetrate the downstream shielding and would be observed in the NOMAD detector via the inverse Primakoff effect, namely the interaction of (pseudo)scalars with virtual photons from the magnetic field of the NOMAD dipole magnet (see Figure 1). The experimental signature of the $a \rightarrow \gamma$ conversion is a single high energy photon which converts in the PRS resulting in a single isolated electromagnetic shower in the ECAL with no activity in any of the other subdetectors.

The probability for the $\gamma \rightarrow a$ or $a \rightarrow \gamma$ coherent conversions is proportional to $\left(\int B_{\perp} d z\right)^{2}$ (see Section 4 ). Therefore we search for axion to photon conversions as an excess of electromagnetic events among interactions occurring in the PRS and ECAL, as they are the most downstream detectors located in the NOMAD magnetic field.

\section{Axion and regenerated photon spectra}

The energy spectra of photons produced in the neutrino target mainly through $\pi^{0}$ decays have been obtained with the same detailed GEANT [30] simulation used to predict the neutrino flux distributions at the NOMAD detector. The effects of pion re-interactions in the target, photon conversion and bremsstrahlung of electrons or positrons have been explicitly taken into account, as well as the correct material composition of the horn for cascade development. Figure 2 shows the energy spectrum of $\pi^{0}$ 's produced in 450 $\mathrm{GeV}$ proton collisions with the neutrino target in a cone of $\sim 2 \mathrm{mrad}$ and the energy spectrum of photons pointing to the NOMAD fiducial area. The fluxes and spectra of $a$ 's from $\gamma \rightarrow a$ conversions in the horn magnetic field, as 
well as those of photons from $a \rightarrow \gamma$ conversions in NOMAD were calculated based on results of ref. [19], [20]. In what follows we consider the axion case for simplicity, but the results are valid also for the scalar case.

In the general case, which includes photon absorption in the target, the probability to find a photon at a distance $L$ from the upstream edge of the NOMAD magnetic field can be calculated from the result of a numerical integration of the $a \rightarrow \gamma$ transition amplitude which is given by the following expression [19],[20]:

$<\gamma(L) \mid a(0)>=\frac{1}{2 M} \exp \left(-\int_{0}^{L} d z \Gamma / 2\right) \int_{0}^{L} d z B_{\perp}(z) \exp \left(i \int_{0}^{z} d z^{\prime}[q-i \Gamma / 2]\right)$

where $M \equiv 1 / g_{a \gamma \gamma}, B_{\perp}$ is the magnetic field component perpendicular to the axion direction. In the above formula, $q=\left(m_{\gamma}^{2}-m_{a}^{2}\right) / 2 E_{a}$ is the difference between the $a$ and the photon momenta in the medium for an axion energy $E_{a} ; m_{\gamma}$ plays the role of the plasma frequency (photon mass) in the NOMAD target medium: $m_{\gamma}^{2}=4 \pi \alpha N_{e} / m_{e}$ with $N_{e}$ the electron number density and $m_{e}$ the electron mass. For the average density of the NOMAD target $m_{\gamma} \approx 7 \mathrm{eV}$. Here, we use $\hbar=c=1$, so $\Gamma \simeq 1 / X_{0} \simeq 3.5 \times 10^{-8} \mathrm{eV}$ is the photon absorption rate in the NOMAD target. The photon absorption in the target results in a reduction of $40 \%$ of the signal. For the case of inverse $\gamma \rightarrow a$ conversion the transition amplitude is given by the same Eq.(3) taking into account that the $a \rightarrow \gamma$ conversion rate is twice that of the inverse process for unpolarized photons with the same coupling strength.

In the absence of photon absorbtion, the absolute maximum of the $a \rightarrow \gamma$ transition probability at a distance $L$ is given by the formula

$$
P_{\gamma}(L)=1.25 \times 10^{-10}\left[\frac{B_{\perp}}{0.4 T}\right]^{2} \cdot\left[\frac{L}{570 \mathrm{~cm}}\right]^{2} \cdot\left[\frac{10^{5} \mathrm{GeV}}{M}\right]^{2}
$$

and corresponds to the case when $|q L| \ll \pi$, i.e. when the axion and the photon fields remain in phase [19],[20] and propagate coherently over the length $L$. For $<E_{a}>\sim 20 \mathrm{GeV}$ and for $L$ equal to the total length of the magnetic field, the axion mass range which can be explored at NOMAD with the photonregeneration method is $m_{a} \lesssim 40 \mathrm{eV}$.

The expected shape of the energy distributions for photons from the $a \rightarrow \gamma$ conversions at the end of the NOMAD detector is shown in Figure 3 for different axion masses. The axion flux from the horn was calculated by integrating Eq.(3) over photon trajectories pointing to the NOMAD fiducial area. It is seen that the photon spectra are quite sensitive to the axion mass. The higher the axion mass the harder is the spectrum needed to fulfil the coherence con- 
dition. Low axion masses tend to contribute mainly to the low energy part of the spectrum. The numbers of axions from the horn and of photons at the PRS detector calculated for a coupling $g_{a \gamma \gamma}=10^{-4} \mathrm{GeV}^{-1}$ and for $1.08 \times 10^{19} \mathrm{pot}$ are shown in Table 1. One can see that for axion masses above $\sim 100 \mathrm{eV}$ the expected number of events at NOMAD drops very fast.

Table 1

Expected numbers of axions and photons $\left(E_{\gamma}>3 \mathrm{GeV}\right)$ obtained using the photonregeneration method calculated for the coupling $g_{a \gamma \gamma}=10^{-4} \mathrm{GeV}^{-1}$ and for $1.08 \times 10^{19}$ pot.

\begin{tabular}{|c|c|c|c|c|}
\hline$m_{a}(\mathrm{eV})$ & 1 & 30 & 100 & 300 \\
\hline axions from Horn & $7.2 \times 10^{9}$ & $6.3 \times 10^{9}$ & $2.8 \times 10^{9}$ & $1.3 \times 10^{8}$ \\
\hline photons at PRS & 46 & 43 & 2.7 & $8.2 \times 10^{-2}$ \\
\hline
\end{tabular}

\section{$5 \quad$ Event samples and selections}

We study events taken with the ECAL trigger during the 1996 run period. The selection criteria for $a \rightarrow \gamma$ events are based on a full Monte Carlo simulation of $a \rightarrow \gamma$ conversions in the NOMAD detector. These criteria are similar to those used in our previous search for a light gauge boson [23]. The difference between the two analyses is due to the lower ECAL energy cut and the additional background simulations described in Section 6 .

Candidate events were identified by the following criteria:

- $\overline{D C} \times \overline{T R D}$ : no significant activity in the DC or TRD as measured by the number of hits in these two detectors.

- $P R S \times E C A L$ : presence of an isolated PRS cluster in both planes matched to an isolated ECAL cluster. The energy of the ECAL cluster was required to be greater than $5 \mathrm{GeV}$ and its shape to fit that expected from an electromagnetic shower[31].

These criteria selected isolated electromagnetic showers in the ECAL that originated from photons converted in the preshower.

- $\overline{H C A L}$ : no energy above $0.4 \mathrm{GeV}$.

- $\overline{M U O N}$ : no track(s) in the muon chambers matched to the PRS cluster.

After imposing the above requirements, we have found 312 candidate events for $a \rightarrow \gamma$ conversion in the initial sample of $2.47 \times 10^{6}$ events recorded with the ECAL trigger. The amount of background from standard neutrino interactions in this sample was evaluated using a Monte Carlo simulation program (see Section 6). 
Applying the $\overline{D C} \times \overline{T R D} \times P R S \times E C A L(>1 G e V) \times M U O N$ selection criteria to the initial sample we identified a sample of $\nu_{\mu} C C$ events collected with the ECAL trigger. Here, MUON denotes a single muon matched to a PRS cluster. This sample corresponds mainly to neutrino interactions occurring in the PRS region defined as the PRS itself and in addition the downstream TRD and upstream ECAL regions. The spectra of energy deposited in the ECAL for these selected $\nu_{\mu} C C$ events and for simulated events which pass the same reconstruction program and selection cuts are in agreement. The ECAL trigger efficiency was obtained from a Monte Carlo simulation and was found to be $71 \%$ and $97 \%$ for $\nu_{\mu} C C$ and $a \rightarrow \gamma$ events, respectively. From the simulation of the neutrino flux, the known cross section for $\nu_{\mu} C C$ interactions and the overall selection efficiency, the number of protons on target giving rise to the observed number of $\nu_{\mu} C C$ events was calculated to be $N_{p o t}=$ $1.08 \times 10^{19}$. This number agrees within $5 \%$ with the value measured by the WANF beam monitors $\left(1.12 \times 10^{19}\right)$ and was used for normalisation. Additional uncertainties arise from the contribution of events from neutrino interactions in the downstream TRD region which pass the $\overline{D C}$ and $\overline{T R D}$ cuts and from backscattering in events occurring in the PRS. By varying the $\overline{D C}$ and $\overline{T R D}$ cuts, it was found that the systematic error on the number of $\nu_{\mu} C C$ events from these effects is smaller than $5 \%$.

\section{$6 \quad$ Background Events}

The relative neutrino beam composition in the NOMAD detector is predicted to be $\nu_{\mu}: \bar{\nu}_{\mu}: \nu_{e}: \bar{\nu}_{e}=1.00: 0.061: 0.0094: 0.0024$, with average energies of 23.5, $19.2,37.1$, and $31.3 \mathrm{GeV}$, respectively [32]. The main background to $a \rightarrow \gamma$ events is expected from neutrino processes with a significant electromagnetic component in the final state and with no significant energy deposition in the HCAL.

The following neutrino processes occurring either in the PRS region or in the upstream region, which includes the magnet coil and DC target, were considered:

- $\nu_{\mu} C C$ interactions classified as muonless because the muon was not detected;

- inclusive $\pi^{0}$ production from $\nu_{\mu}$ neutral current (NC) interactions;

- coherent and diffractive $\pi^{0}$ production;

- quasi-elastic $\nu_{e}$ scattering;

- $\nu_{e} C C$ and $\bar{\nu}_{e} C C$ interactions.

To evaluate the amount of background in the data sample, simulated events were processed through the same reconstruction program and selection criteria used for real data. All background distributions were then summed up, tak- 
ing into account the corresponding normalisation factors. These factors were calculated from beam composition and cross-sections for the processes listed above. The total number of expected candidate events after applying the selection criteria are given in Table 2 for each background component. The total background in the data sample was estimated to be $272 \pm 18$ events, where statistical and systematic errors were added in quadrature. The fraction of neutrino interactions satisfying all cuts is about $7.5 \times 10^{-4}$.

Table 2

Background Estimate. Total number of interactions in the PRS region and in the upstream region (last two columns) and the resulting number of expected background candidate events.

\begin{tabular}{|c|c|c|c|c|c|c||c|c|}
\hline item & $\nu_{\mu} C C$ & $\nu_{\mu} N C$ & $\nu_{e} C C$ & $\nu_{e} Q E L$ & $\bar{\nu}_{e} C C$ & Coh. $\pi^{0}$ & $\nu_{\mu} N C$ & Coh. $\pi^{0}$ \\
\hline \hline $\begin{array}{c}\text { Total number } \\
\text { of interactions }\end{array}$ & 274250 & 81328 & 4114 & 105 & 439 & 603 & 451376 & 3670 \\
\hline $\begin{array}{c}\text { Number of } \\
\text { expected } \\
\text { candidates }\end{array}$ & $5.9 \pm 3.5$ & $96.9 \pm 9.6$ & $85.1 \pm 10.9$ & $16.4 \pm 1.9$ & $11.7 \pm 1.8$ & $38.6 \pm 8.4$ & $5.2 \pm 2.3$ & $11.8 \pm 3.4$ \\
\hline
\end{tabular}

\section{$7 \quad$ Results}

Figure 4 shows the overall background and candidate event energy spectra in the ECAL. The agreement between data and Monte Carlo is reasonable. The overall efficiency for single high energy photon detection in the PRS/ECAL was found to be $\sim 25 \%$ for axion masses $m_{a} \lesssim 40 \mathrm{eV}$. The inefficiency is mostly due to the requirement of photon conversion in the PRS, the ECAL energy cut and PRS/ECAL matching.

By subtracting the number of expected background events from the number of candidate events we obtain $N_{a \rightarrow \gamma}=40 \pm 25$ events showing no significant excess of $a \rightarrow \gamma$ conversion-like events. The $90 \% C L$ upper limit for the coupling $g_{a \gamma \gamma}$ was calculated by using the following scaling relations:

$$
N_{a \rightarrow \gamma}^{M=10^{4}} \mathrm{GeV}=\int_{E_{1}}^{E_{2}} \Phi_{\gamma} \cdot P_{\gamma \rightarrow a}\left(m_{a}, E_{\gamma}\right) \cdot P_{a \rightarrow \gamma}\left(m_{a}, E_{\gamma}\right) \cdot \varepsilon_{s e l} \cdot N_{p o t} \cdot d E_{\gamma}
$$

and

$$
g_{a \gamma \gamma}^{90 \% C L}<10^{-4} \mathrm{GeV}^{-1} \times\left(\frac{N_{a \rightarrow \gamma}^{90 \%}}{N_{a \rightarrow \gamma}^{M=10^{4} G e V}}\right)^{1 / 4}
$$

where $N_{a \rightarrow \gamma}^{M=10^{4} \mathrm{GeV}}$ is the number of expected candidate events calculated for a coupling $g_{a \gamma \gamma}=10^{-4} \mathrm{GeV}^{-1}$ in the energy region from $E_{1}$ to $E_{2}$ corresponding 
to the signal from the axion of mass $m_{a}, N_{a \rightarrow \gamma}^{90 \%}$ is the $90 \% C L$ upper limit for the expected number of signal events in the same energy region, $\varepsilon_{\text {sel }}$ is the selection efficiency, which was found to be practically independent of the photon energy $E_{\gamma}\left(\right.$ for $\left.E_{\gamma}>5 G e V\right), \Phi_{\gamma}, P_{\gamma \rightarrow a}\left(m_{a}, E_{\gamma}\right), P_{a \rightarrow \gamma}\left(m_{a}, E_{\gamma}\right)$ are the flux of photons per pot, probability of photon to axion conversion in the horn magnetic field and probability of axion to photon conversion in the NOMAD magnetic field, respectively.

The region excluded in the $\left(m_{a}, g_{a \gamma \gamma}\right)$ plane at $90 \% C L$ is shown in Figure 5 together with the results of other experiments. For the mass region $m_{a} \lesssim 40 \mathrm{eV}$ the limit is

$$
g_{a \gamma \gamma}<1.5 \times 10^{-4} \mathrm{GeV}^{-1}
$$

and is valid for both scalar and (pseudo)scalar cases. The statistical limit on the sensitivity is set by the values of $\int B_{\perp} d z$ in the horn and the NOMAD detector. The uncertainties on $\pi^{0}$ production rate $(\simeq 20 \%)$ and neutrino fluxes ( $\lesssim 10 \%$ ), and on some cross-sections for neutrino interaction in the PRS (e.g. coherent $\pi^{0}$ production) and backscattering effects give a large contribution to the systematic error. Since the sensitivity of this search is proportional to $g_{a \gamma \gamma}^{4}$, the upper limit from Eq.(6) depends weakly on variations of the selected photon energy interval. The limit of Eq.(7) is approximately a factor of 10 better than the best direct limit extracted from the recently published limit on $\operatorname{Br}(\Upsilon \rightarrow \gamma a)$ obtained by the CLEO collaboration [13] .

The limit of Eq.(7) is valid for a (pseudo)scalar lifetime $\tau_{a}[s]>10^{-15} m_{a}[\mathrm{eV}]$ which is much shorter than the decay time of a standard axion into two photons in the above mass range. The attenuation of the $a$ flux due to interactions in the shielding was found to be negligible, since for the limit of Eq.(7) the axion mean free path in iron is $\geq 10^{4} \mathrm{~km}$, as compared with the iron and earth shielding total length of $0.4 \mathrm{~km}$ used in our beam.

\section{Acknowledgements}

We gratefully acknowledge the CERN SPS accelerator and beam-line staff for the magnificent performance of the neutrino beam. The experiment was supported by the following funding agencies: Australian Research Council (ARC) and Department of Industry, Science, and Resources (DISR), Australia; Institut National de Physique Nucléaire et Physique des Particules (IN2P3), Commissariat à l'Energie Atomique (CEA), France; Bundesministerium für Bildung und Forschung (BMBF, contract 05 6DO52), Germany; Istituto Nazionale di Fisica Nucleare (INFN), Italy; Russian Foundation for Basic Research, Institute for Nuclear Research of the Russian Academy of Sciences, Russia; Fonds National Suisse de la Recherche Scientifique, Switzerland; 
Department of Energy, National Science Foundation (grant PHY-9526278), the Sloan and the Cottrell Foundations, USA. F.J.P. Soler is supported by a TMR Fellowship from the European Commission. We thank Prof. V.Matveev and I.Tkachev for many interesting talks and comments. Discussions with G.Raffelt on axion to photon conversion in a medium are gratefully acknowledged.

\section{References}

[1] C. Caso et al., Review of Particle Properties, Europ. Phys. Journ. C3 (1998) 1.

[2] S. Weinberg, Phys. Rev. Lett. 40 (1978) 223;

F. Wilczek, Phys. Rev. Lett. 40 (1978) 279.

[3] R.D. Peccei and H. Quinn, Phys. Rev. Lett. 38 (1977) 1440.

[4] M. Dine, W. Fischler and M. Srednicki, Phys. Lett. B104 (1981) 199;

A. Zhitnitski, Yad. Fiz. 31 (1980) 497, (Sov. Journ. Nucl. Phys. 31 (1980) 260).

[5] J.E. Kim, Phys. Rev. Lett. 43 (1979) 103;

M. Schifman, A. Vainstein and V. Zakharov, Nucl. Phys. 166 (1981) 493.

[6] N.V. Krasnikov, V.A. Matveev and A.N. Tavkhelidze, Sov. Journ. Part. Nucl. 12 (1981) 38;

J.E. Kim, Phys. Rep. 150 (1987) 1;

H.Y. Cheng, Phys. Rep. 158 (1988) 1;

G.G Raffelt, Phys. Rep. 198 (1990) 1.

[7] P. Horava and E. Witten, Nucl. Phys. B460 (1996) 506;

Nucl. Phys. B475 (1996) 94.

[8] N. Arkani-Hamed, S. Dimopoulos and G. Dvali, Phys. Lett. B429 (1998) 263;

I. Antoniadis et al., Phys. Lett. B436 (1998) 257.

[9] G. Ruoso et al., Z. Phys. C56 (1992) 505.

[10] R. Cameron et al., Phys. Rev. D47 (1993) 3707.

[11] M.S. Alam et al., Phys. Rev. D27 (1983) 1665; N.J. Baker et al., Phys. Rev. Lett. 59 (1987) 2832.

[12] U. Amaldi, G. Carboni, B. Jonson, and J. Thun, Phys. Lett. B153 (1985) 444;

S. Orito et al., Phys. Rev. Lett. 63 (1989) 597;

M.V. Akopian,G.S. Atoyan, S.N. Gninenko, and V.V. Sukhov, Phys. Lett. B272 (1991) 443;

S.N. Gninenko, Yu.M. Klubakov, A.A. Poblaguev, and V.E. Postoev, Phys. Lett. B237 (1990) 287; 
T. Maeno et al., Phys. Lett. B351 (1995) 574;

S. Asai, S. Orito, K. Yoshimura, and T. Haga, Phys. Rev. Lett. 66 (1991) 2440.

[13] R. Balest et al., CLEO Collaboration, Phys. Rev. D51 (1995) 2053.

[14] S.N. Gninenko et al.," Search for light (pseudo)scalar penetrating particles in the SPS beam", NOMAD Int. Note/99-004 (unpublished).

[15] G.G. Raffelt, Preprint hep-ph/9903472,

to appear in Ann. Rev. Nucl. Part. Sc. Vol.49 (1999); Preprint hep-ph/9806506.

[16] S.I. Blinnikov and M.I. Visotsky, Yad. Fiz. 52 (1990) 544;

Sov. J. Nucl. Phys. 52 (1990) 348.

[17] E. Iacopini and E.Zavattini, Phys. Lett. B85 (1979) 151.

[18] L. Maiani, R. Petronzio and E.Zavattini, Phys. Lett. B175 (1986) 359.

[19] G. Raffelt and L. Stodolsky, Phys. Rev. D37 (1988) 1237.

[20] K. Van Bibber et al., Phys. Rev. D39 (1989) 2089.

[21] P. Sikivie, Phys. Rev. Lett. 51 (1983) 1415; idid 52 (1984) 695.

[22] A.A. Anselm, Yad. Fiz. 42 (1985) 1480, Sov. Journ. Nucl. Phys. 42 (1985) 936;

K. Van Bibber et al., Phys. Rev. Lett. 59 (1987) 759 .

[23] J. Altegoer et al., NOMAD Collaboration, Phys. Lett B428 (1998) 197.

[24] G. Acquistapace et al., CERN-ECP/95-14, July 95.

[25] J. Altegoer et al., NOMAD Collaboration, Nucl. Instr. and Meth. A404 (1998) 96.

[26] M. Anfreville et al., "The drift chambers of the NOMAD detector", paper in preparation.

[27] G. Bassompierre et al., Nucl. Instr. and Meth. A403 (1998) 363;

G. Bassompierre et al., Nucl. Instr. and Meth. A411 (1998) 63.

[28] D. Autiero et al., Nucl. Instr. and Meth. A372 (1996) 556;

D. Autiero et al., Nucl. Instr. and Meth. A373 (1996) 358;

D. Autiero et al., Nucl. Instr. and Meth. A387 (1997) 352;

D. Autiero et al., Nucl. Instr. and Meth. A411 (1998) 285.

[29] J. Altegoer et al., Nucl. Instr. and Meth. A428 (1999) 299.

[30] GEANT: Detector description and simulation tool, CERN Programming Library Long Writeup W5013.

[31] R. Petti, PhD thesis, University of Pavia 1998;

D. Autiero et al., Nucl. Instr. and Meth. A425 (1999) 190.

[32] G. Collazuol et al., presented at NOW98 Workshop, Amsterdam, 7-9 September 1998, CERN Preprint OPEN-98-032. 

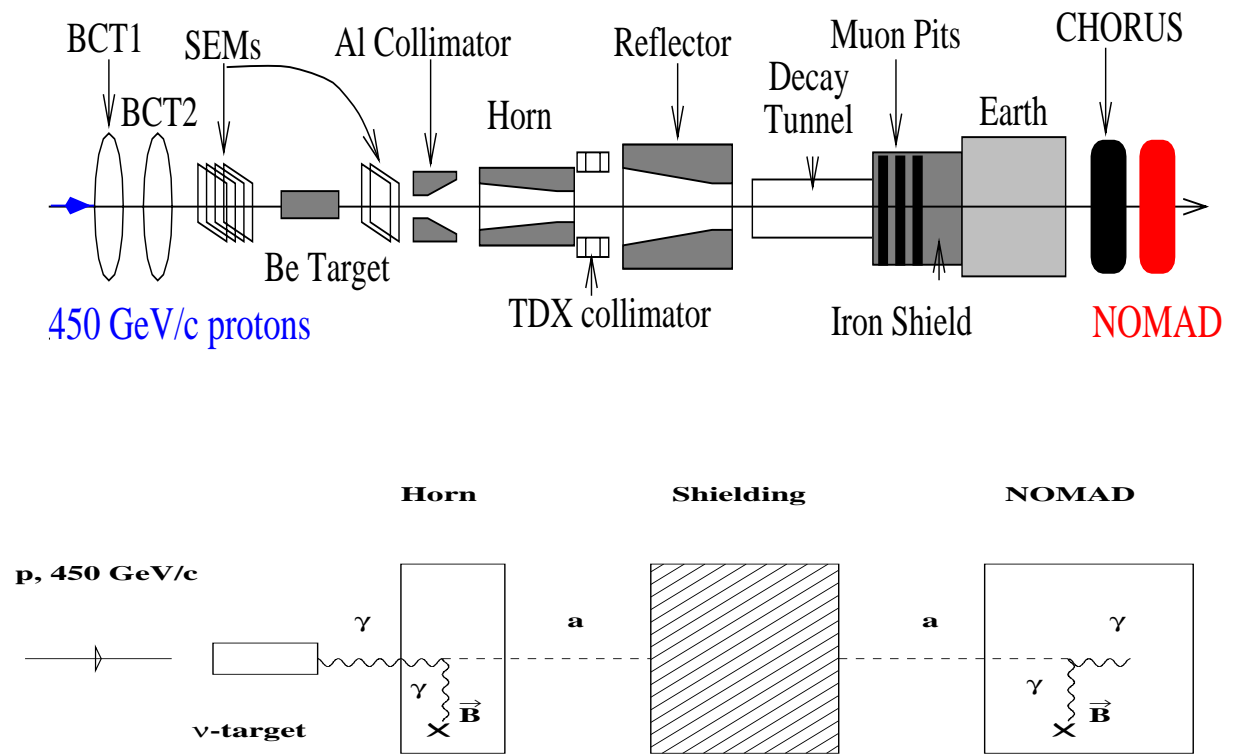

Fig. 1. Schematic layout of the WANF beam line (not to scale) and an illustration of the principle of the WANF-NOMAD high energy photon-regeneration experiment.
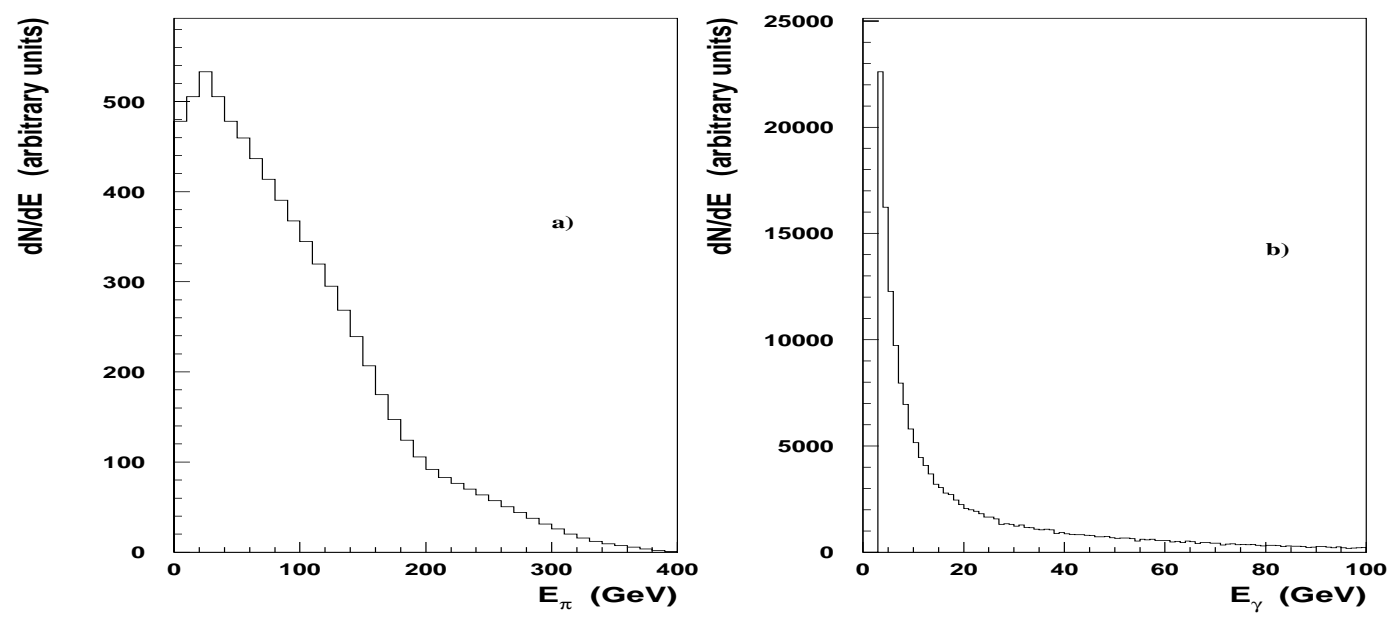

Fig. 2. a) The energy spectrum of $\pi^{0}$ 's produced in $450 \mathrm{GeV}$ proton collisions with the neutrino target in a cone of $\sim 2$ mrad. b) Energy spectrum of the photons crossing the horn magnetic field and momenta pointing towards the NOMAD fiducial area. 

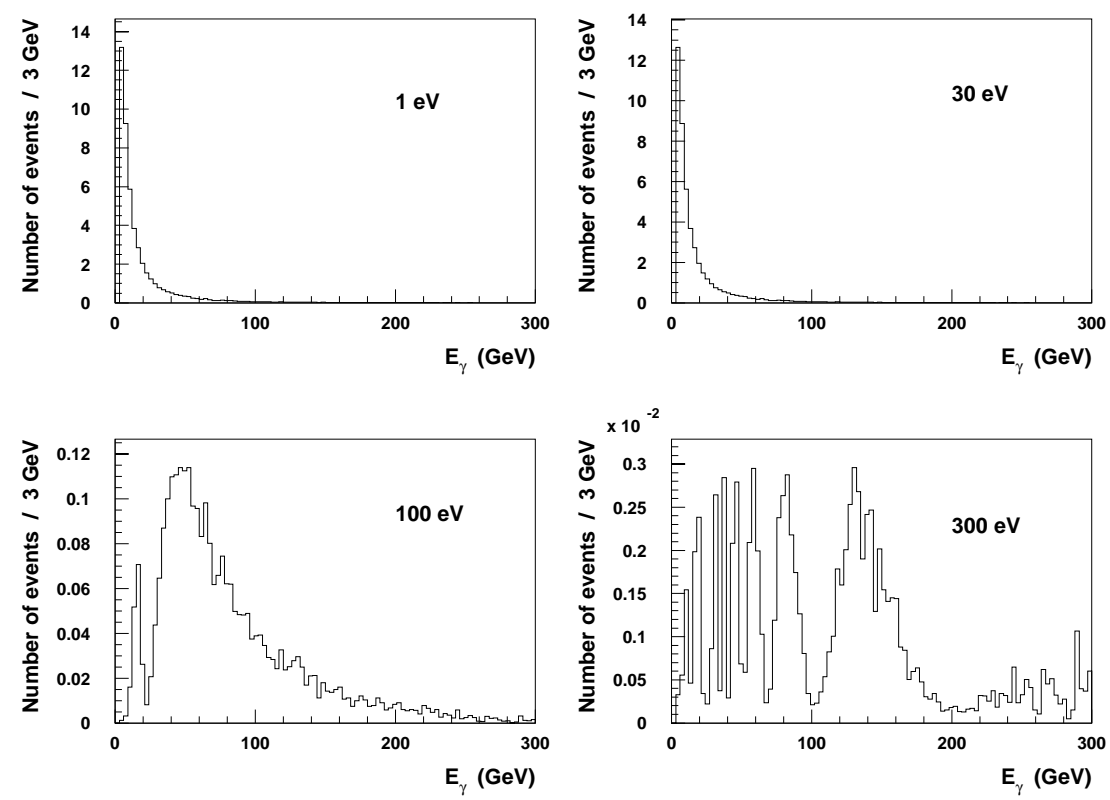

Fig. 3. Energy spectra of photons entering the PRS, as expected from $a \rightarrow \gamma$ conversions of axions produced through $\gamma \rightarrow$ a conversions in the horn magnetic field. The effect of the axion mass on the energy spectra is clearly seen. The calculations are performed for $1.08 \times 10^{19}$ pot and $g_{a \gamma \gamma}=10^{-4} \mathrm{GeV}^{-1}$. 


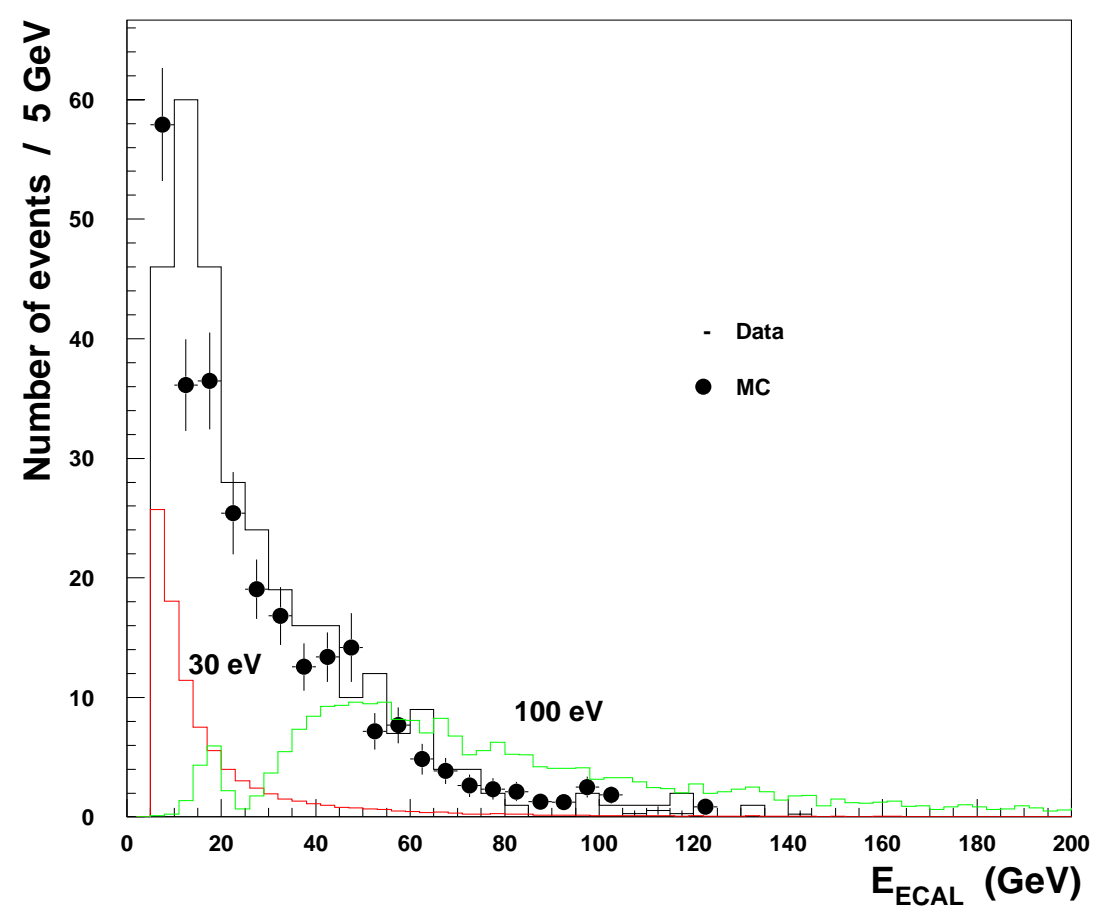

Fig. 4. The ECAL energy spectra for candidate (solid line) and background (full circles) events. Systematic errors are not shown. The shape of the axion signals corresponding to masses $m_{a}=30 \mathrm{eV}$ and $m_{a}=100 \mathrm{eV}$ are shown for comparison. 


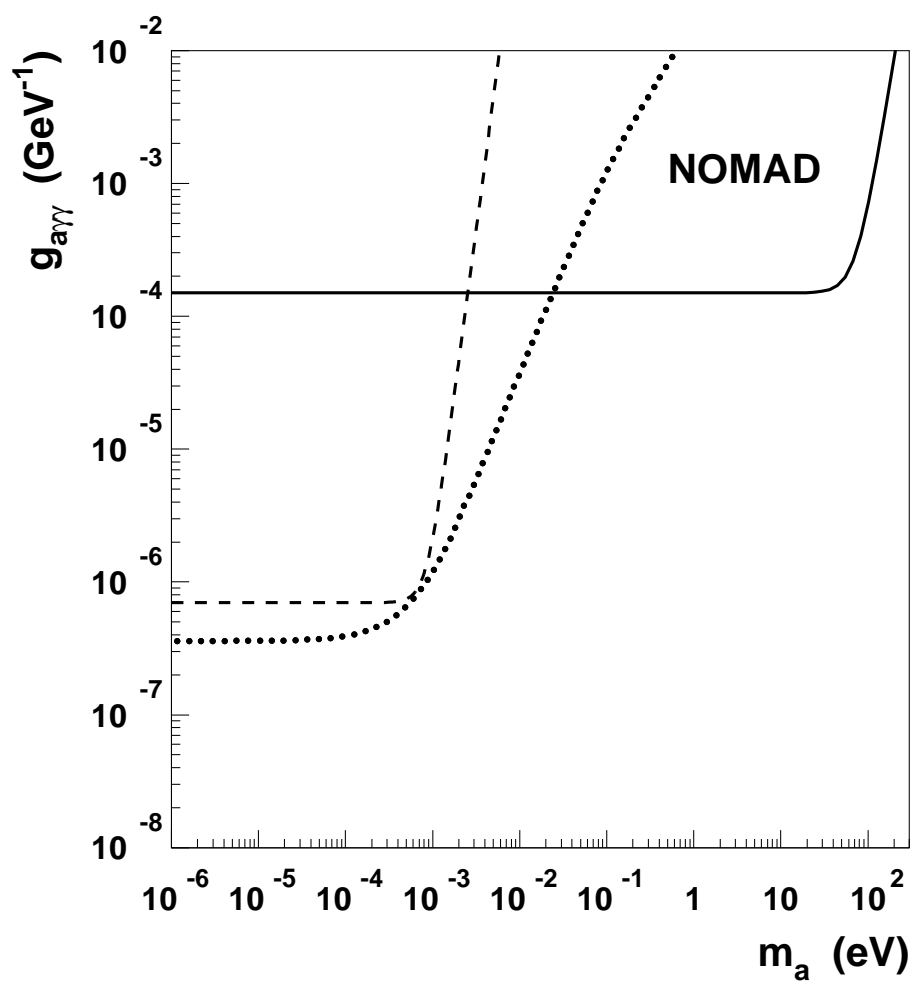

Fig. 5. Upper limit on the coupling $g_{a \gamma \gamma}$ as a function of the (pseudo)scalar mass $m_{a}$ derived from the present analysis and from the direct searches of light (pseudo)scalars performed by using the polarisation rotation of a laser beam in a magnetic field [10](dotted line) and using the laser photon regeneration method [9], [10] (dashed line). 